\title{
KRULL DIMENSION AND REGULARITY OF BINOMIAL EDGE IDEALS OF BLOCK GRAPHS
}

\author{
CARLA MASCIA AND GIANCARLO RINALDO
}

\begin{abstract}
We give a lower bound for the Castelnuovo-Mumford regularity of binomial edge ideals of block graphs by computing the two distinguished extremal Betti numbers of a new family of block graphs, called flower graphs. Moreover, we present linear time algorithms to compute the CastelnuovoMumford regularity and the Krull dimension of binomial edge ideals of block graphs.
\end{abstract}

\section{INTRODUCTION}

In 2010, binomial edge ideals were introduced in 8 and also appeared independently in [15]. Let $S=K\left[x_{i}, y_{j}\right]_{1 \leq i, j \leq n}$ be the polynomial ring in $2 n$ variables with coefficients in a field $K$. Let $G$ be a graph on vertex set $[n]$ and edges $E(G)$. The ideal $J_{G}$ of $S$ generated by the binomials $f_{i j}=x_{i} y_{j}-x_{j} y_{i}$ such that $i<j$ and $\{i, j\} \in E(G)$ is called the binomial edge ideal of $G$. Any ideal generated by a set of 2 -minors of a $2 \times n$-matrix of indeterminates may be viewed as the binomial edge ideal of a graph.

For a set $T \subset[n]$, let $G_{[n] \backslash T}$ be the induced subgraph of $G$ with vertex set $[n] \backslash T$ and $G_{1}, \ldots, G_{c(T)}$ the connected components of $G_{[n] \backslash T}$. T is a cutset of $G$ if $c(T \backslash\{i\})<c(T)$ for each $i \in T$, and we denote by $\mathcal{C}(G)$ the set of all cutsets for $G$. In 8 and 15 the authors gave a description of the primary decomposition of $J_{G}$ in terms of prime ideals induced by the set $\mathcal{C}(G)$ (see (2)). Thanks to this result the following formula for the Krull dimension is obtained

$$
\operatorname{dim} S / J_{G}=\max _{T \in \mathcal{C}(G)}\{n+c(T)-|T|\} .
$$

The second author in [17] described an algorithm to compute the primary decomposition (2), and hence the Krull dimension. Unfortunately, this algorithm is exponential in time and space.

A block graph, also known as clique tree, is a graph whose blocks are cliques. In general, computing the depth of a ring is a difficult task. In [4] the authors prove that when $G$ is a block graph, depth $S / J_{G}=n+c$ and, equivalently, proj $\operatorname{dim} S / J_{G}=$ $n-c$, where $c$ is the number of connected components of $G$. Given any block graph, such an immediate formula for the Krull dimension does not exist, and we believe that is hard to obtain something that is better than (11).

Nevertheless we believe the block graphs are the easier but not trivial class where we can obtain a good algorithm to compute the Krull dimension. We present an algorithm (Theorem 2.5) that is linear in time and space and computes the Krull dimension. The idea is to find a minimal prime ideal of minimum height since it induces the Krull dimension of $S / J_{G}$. We have implemented the algorithm using CoCoA (2]), when $G$ is a tree and it is freely downloadable on [13. 
Another fundamental invariant that has been studied in deep is the CastelnuovoMumford regularity of binomial edge ideal. Lower and upper bounds for the regularity are known by Matsuda and Murai [14] and Kiani and Saeedi Madani [12]. In [5], the authors proved the conjecture posed in 20] for closed graphs and block graphs. For these graphs, the regularity of $S / J_{G}$ is bounded below by the length of the longest induced path of $G$ and above by $c(G)$, where $c(G)$ is the number of maximal cliques of $G$. Furthermore, Kiani and Saeedi Madani characterized all graphs whose binomial edge ideal have regularity 2 and regularity 3, see [19] and [21.

It is still an open problem to determine an explicit formula for the regularity of binomial edge ideals for block graphs in terms of the combinatorics of the graph. Recently, in [9] Herzog and the second author computed one of the distinguished extremal Betti number of the binomial edge ideal of a block graph and classify all block graphs admitting precisely one extremal Betti number giving a natural lower bound for the regularity of any block graph. Jayanthan et al in [10] and in [11] obtained a related result for trees, a subclass of block graphs.

Inspired by these results we define a new class of graphs, namely the flower graphs (see Definition 3.1 and Figure 11), for which we compute the superextremal Betti numbers (see Theorem 3.4) and the regularity (see Corollary 3.5). As a consequence we obtain new lower bounds in Theorem 3.6 and Corollary 3.8 for the regularity of any block graph.

In Section 4, we state the main result of this work, Theorem 4.2, that provides an efficient method to compute the Castelnuovo-Mumford regularity of any binomial edge ideal of block graphs, exploiting the notion of end-flowers (see Definition 4.1) and by means of an unique block graph traversal.

\section{On the height of Minimal PRIME IDEALS OF $J_{G}$ AND DECOMPOSABILITY OF BLOCK GRAPHS}

We start this section by recalling the formula to compute the primary decomposition of a binomial edge ideal $J_{G}$. Let $G$ be a graph on $[n], T \subset[n]$ is called cutset of $G$ if $c(T \backslash\{i\})<c(T)$ for each $i \in T$, where $c(T)$ denotes the number of connected components induced by removing $T$ from $G$. We denote by $\mathcal{C}(G)$ the set of all cutsets of $G$. When $T \in \mathcal{C}(G)$ consists of one vertex $v, v$ is called a cutpoint. Define

$$
P_{T}(G)=\left(\bigcup_{i \in T}\left\{x_{i}, y_{i}\right\}, J_{\tilde{G}_{1}}, \ldots, J_{\tilde{G}_{c(T)}}\right) \subseteq S
$$

where $\tilde{G}_{i}$, for $i=1, \ldots, c(T)$, denotes the complete graph on $V\left(G_{i}\right) . P_{T}(G)$ is a prime ideal of height $n-c(T)+|T|$. It holds

$$
J_{G}=\bigcap_{T \in \mathcal{C}(G)} P_{T}(G) .
$$

We denote by $\mathcal{M}(G)$ the minimal prime ideals of $J_{G}$, by $\mathcal{M i n h}(G) \subseteq \mathcal{M}(G)$ the minimal prime ideals $P_{T}(G)$ of minimum height and by $\mathcal{M a x h}(G) \subseteq \mathcal{M}(G)$ the minimal prime ideals $P_{T}(G)$ of maximum height.

A subset $C$ of $V(G)$ is called a clique of $G$ if for all $i, j \in C$, with $i \neq j$, one has $\{i, j\} \in E(G)$. A maximal clique is a clique that cannot be extended by including one more adjacent vertex. 
A connected subgraph of $G$ that has no cutpoint and is maximal with respect to this property is a block. $G$ is called block graph if all its blocks are complete graphs. One can see that a graph $G$ is a block graph if and only if it is a chordal graph in which every two maximal cliques have at most one vertex in common. Let $G$ be a block graph, an endblock of $G$ is a block having exactly one cutpoint.

The clique degree of $v$, denoted by $\operatorname{cdeg}(v)$, is the number of maximal cliques to which $v$ belongs. A vertex $v$ is called a free vertex of $G$ if $\operatorname{cdeg}(v)=1$, and is called an inner vertex of $G$ if $\operatorname{cdeg}(v)>1$.

Definition 1.1 A graph $G$ is decomposable if exists a decomposition

$$
G=G_{1} \cup G_{2}
$$

with $V\left(G_{1}\right) \cap V\left(G_{2}\right)=\{v\}$ such that $v$ is a free vertex of $G_{1}$ and $G_{2}$. If $G$ is not decomposable, we call it indecomposable. By a recursive decomposition (3) applied to each $G_{1}$ and $G_{2}$, after a finite number of steps we obtain

$$
G=G_{1} \cup \cdots \cup G_{r}
$$

where $G_{1}, \ldots, G_{r}$ are indecomposable and for $1 \leq i<j \leq r$ either $V\left(G_{i}\right) \cap V\left(G_{j}\right)=$ $\emptyset$ or $V\left(G_{i}\right) \cap V\left(G_{j}\right)=\left\{v_{i j}\right\}$, where $v_{i j}$ is a free vertex of $G_{i}$ and $G_{j}$. The decomposition (4) is unique up to ordering and we say that $G$ is decomposable into indecomposable graphs $G_{1}, \ldots, G_{r}$.

For the sake of completeness, we collect in the next proposition the results showed in [9] and [16] concerning Krull dimension of $S / J_{G}$, height of the ideals $P_{T}(G)$ and the Castelnuovo-Mumford regularity of $S / J_{G}$, when $G$ is decomposable.

Proposition 1.2 Let $G$ be a graph decomposable into $G_{1}$ and $G_{2}$, with $V\left(G_{1}\right) \cap$ $V\left(G_{2}\right)=\{v\}$. Then

(i) $\operatorname{dim} S / J_{G}=\operatorname{dim} S_{1} / J_{G_{1}}+\operatorname{dim} S_{2} / J_{G_{2}}-2$, where $S_{i}=K\left[x_{j}, y_{j}\right]_{j \in V\left(G_{i}\right)}$ for $i=1,2$;

(ii) height $P_{T}(G)=$ height $P_{T_{1}}\left(G_{1}\right)+$ height $P_{T_{2}}\left(G_{2}\right)$, with $T \in \mathcal{C}(G), T_{1} \in$ $\mathcal{C}\left(G_{1}\right)$, and $T_{2} \in \mathcal{C}\left(G_{2}\right)$ and either $T=T_{1} \cup T_{2}$ or $T=T_{1} \cup T_{2} \cup\{v\}$;

(iii) $\operatorname{reg} S / J_{G}=\operatorname{reg} S / J_{G_{1}}+\operatorname{reg} S / J_{G_{2}}$.

For a block graph $G$, being decomposable can be read from the primary decomposition of $J_{G}$, in particular from the ideals in $\operatorname{Maxh}(G)$.

Proposition 1.3 Let $G$ be a block graph. The following are equivalent:

(i) $G$ is indecomposable;

(ii) if $v \in V(G)$, then $\operatorname{cdeg}(v) \neq 2$;

(iii) $\mathcal{M a x h}(G)=\left\{P_{\emptyset}(G)\right\}$.

Proof.

(1) $\Leftrightarrow(2)$ It is trivial.

$(2) \Rightarrow(3)$ Without loss of generality, let $G$ be connected. Since height $P_{\emptyset}(G)=$ $n-1$, we want to prove that for any $T \neq \emptyset$, height $P_{T}(G)<n-1$. Let $T \in \mathcal{C}(G)$, with height $P_{T}(G) \geq n-1$, that is $c(T)-|T| \leq 1$. If $T=\{v\}$, then $c(T) \leq 2$ or equivalently $\operatorname{cdeg}(v) \leq 2$. Since $v$ is a cutpoint, it is 
not a free vertex, and then $\operatorname{cdeg}(v)=2$, which is in contradiction to the hypothesis. Let $T=\left\{v_{1}, \ldots, v_{r}\right\}$, with $r \geq 2$, such that height $P_{T}(G) \geq$ $n-1$ and suppose it is minimal with respect to this property. In a block graph, $T_{1}=T \backslash\left\{v_{r}\right\}$ is a cutset, too. By definition, $c\left(T_{1}\right)<c(T)$ and $\left|T_{1}\right|=|T|-1$, then $c\left(T_{1}\right)-\left|T_{1}\right|<2$. It follows that height $P_{T_{1}}(G) \geq n-1$, but it is in contradiction to the hypothesis on the minimality of $T$.

$(3) \Rightarrow(2)$ Assume that there exists a vertex $v \in V(G) \operatorname{such}$ that $\operatorname{cdeg}(v)=2$. Let $T=\{v\}$, then height $P_{T}(G)=$ height $P_{\emptyset}(G)=n-1$. Hence, $P_{T}(G) \in$ $\operatorname{Maxh}(G)$, too. The latter is in contradiction to the hypothesis.

We observe that for a generic graph $G$, is not true that if $G$ is indecomposable then $\operatorname{cdeg}(v) \neq 2$ for any $v \in V(G)$. It is sufficient to consider $G=C_{4}$, with $V(G)=\{1, \ldots, 4\}$ and $E(G)=\{\{i, i+1\} \mid i=1, \ldots, 3\} \cup\{1,4\}$. All its vertices have clique degree equal to 2 , but $G$ is indecomposable. Moreover, for a generic graph $G$ being indecomposable is not equivalent to the fact that $P_{\emptyset}(G)$ is the prime ideal of the maximum height in the primary decomposition of $J_{G}$. In fact, consider again $G=C_{4}$. The subset $T=\{1,3\}$ is a cutset for $G$ and height $P_{T}(G)=4$, whereas height $P_{\emptyset}(G)=3$.

\section{KRULL Dimension of BINOMIAL EDGE IDEALS OF BLOCK GRAPHS}

If $G$ is any graph with $n$ vertices, the Krull dimension of $S / J_{G}$ is given by $\operatorname{dim} S / J_{G}=\max _{T \in \mathcal{C}(G)}\{n+c(T)-|T|\}$, and then, in general, to compute it one must investigate all the possible cutsets of $G$. For some classes of graphs, there exists an immediate way to compute the Krull dimension. For example, if $G$ is a complete graph or a graph obtained by gluing free vertices of complete graphs and such that any vertex $v \in V(G)$ is either a free vertex or has $\operatorname{cdeg}(v)=2$, then $\operatorname{dim} S / J_{G}=n+1$. For a generic block graph $G$, we show an algorithm to compute the Krull dimension of $S / J_{G}$ in linear time.

From now on, we consider only connected block graphs, since the Krull dimension of $S / J_{G}$, where $G$ is a graph with $c$ connected components, $G_{1}, \ldots, G_{c}$, is given by the sum of the Krull dimension of $S_{i} / J_{G_{i}}$, with $i=1, \ldots, c$ and $S_{i}=K\left[x_{j}, y_{j}\right]_{j \in V\left(G_{i}\right)}$. Before showing the aforementioned algorithm, we need some auxiliary results.

Lemma 2.1 Let $G$ be a block graph, $P_{T}(G) \in \operatorname{Minh}(G)$, and $v \in V(G)$. If $v$ belongs to

(i) exactly two endblocks, then $P_{T \cup\{v\}}(G) \in \mathcal{M i n h}(G)$;

(ii) at least three endblocks, then $v \in T$.

Proof. Let $P_{T}(G) \in \mathcal{M i n h}(G)$ and let $v$ belong to $r$ endblocks, $B_{1}, \ldots, B_{r}$, with $r \geq$ 2 , and let $G_{1}, \ldots, G_{c}$ be the connected components of $G_{[n] \backslash T}$, then height $P_{T}(G)=$ $n-c+|T|$. Suppose that $v \notin T$. Without loss of generality, we can suppose $v \in G_{1}$. The connected components induced by $T \cup\{v\}$ are $B_{1}^{\prime}, \ldots, B_{r}^{\prime}, G_{1}^{\prime}, G_{2}, \ldots, G_{c}$, where $B_{i}^{\prime}=B_{i} \backslash\{v\}$ for $i=1, \ldots, r$ and $G_{1}^{\prime}=G_{1} \backslash\left\{B_{1}, \ldots, B_{r}\right\}$. If $r=2$ and $G_{1}^{\prime}=\emptyset$, then height $P_{T \cup\{v\}}(G)=\operatorname{height} P_{T}(G)$, and then also $P_{T \cup\{v\}}(G) \in \mathcal{M i n h}(G)$. If $r \geq 3$ or $r=2$ and $G_{1}^{\prime} \neq \emptyset$, the number of connected components induced by $T \cup\{v\}$ is at least $r+c$ and hence it is greater than or equal to $c+2$. Thus, 
height $P_{T \cup\{v\}}(G) \leq n-(c+2)+(|T|+1)<$ height $P_{T}(G)$, which is in contradiction to the minimality of $P_{T}(G)$.

Remark 2.2. Let $G$ be a block graph and $T \in \mathcal{C}(G)$ such that $P_{T}(G) \in \mathcal{M i n h}(G)$. If $\left\{v_{1}, \ldots, v_{r}\right\} \subseteq T$ is the set of all the vertices in $T$ with clique degree equal to 2, by Proposition $1.2(2), P_{T \backslash\left\{v_{1}, \ldots, v_{r}\right\}}(G) \in \mathcal{M i n h}(G)$.

Lemma 2.3 Let $G$ be a block graph and $v \in V(G)$ be a cutpoint. If

(i) $v$ belongs to at least 2 endblocks of an indecomposable component of $G$,

(ii) $P_{T^{\prime}}(H) \in \mathcal{M i n h}(H)$, where $T^{\prime} \in \mathcal{C}(H)$ and $H$ is the graph obtained from $G$ by removing $v$ and the endblocks to which $v$ belongs

then $P_{T^{\prime} \cup\{v\}}(G) \in \mathcal{M i n h}(G)$.

Proof. Let $T \in \mathcal{C}(G)$ be such that $P_{T}(G) \in \mathcal{M i n h}(G)$ and $v \in T$. By Lemma 2.1, we know that such $T$ exists. Let $T=T_{1} \cup\{v\}$. Let $r \geq 2$ be the number of endblocks to which $v$ belongs, then $c(T)=r+c\left(T_{1}\right)$, where $c\left(T_{1}\right)$ denotes the number of connected components of $H$ induced by $T_{1}$. It follows that

$$
\text { height } \begin{aligned}
P_{T}(G) & =n-\left(r+c\left(T_{1}\right)\right)+\left(1+\left|T_{1}\right|\right) \\
& =n-V(H)-r+1+\left[V(H)-c\left(T_{1}\right)+\left|T_{1}\right|\right] \\
& =s+\operatorname{height} P_{T_{1}}(H)
\end{aligned}
$$

where $s=n-V(H)-r+1$. Observe that $P_{T_{1}}(H) \in \mathcal{M i n h}(H)$ : if there exists $T_{2} \in$ $\mathcal{C}(G)$ such that height $P_{T_{2}}(H)<$ height $P_{T_{1}}(H)$, then height $P_{T_{2} \cup\{v\}}(G)$ is lower than height $P_{T}(G)$, and this is in contradiction to the minimality of $P_{T}(G)$. Since, by hypothesis, $P_{T^{\prime}}(H), P_{T_{1}}(H)$ have the same height, it follows height $P_{T}(G)=$ $s+$ height $P_{T^{\prime}}(H)=$ height $P_{T^{\prime} \cup\{v\}}(G)$, and $P_{T^{\prime} \cup\{v\}}(G) \in \mathcal{M i n h}(G)$.

The following result is the core of the algorithm that allows to compute the Krull dimension of $S / J_{G}$.

Theorem 2.4 Let $G$ be a block graph and $T=\left\{v_{1}, \ldots, v_{t}\right\} \in \mathcal{C}(G)$. We denote by $H_{0}$ the graph $G$ and by $H_{i}$ the graph obtained from $H_{i-1}$ by removing $v_{i}$ and the endblocks to which $v_{i}$ belongs, for all $i=1, \ldots, t$. If

(i) $v_{i}$ belongs to at least 2 endblocks of an indecomposable component of $H_{i-1}$, for all $i=1, \ldots, t$,

(ii) $H_{t}$ is decomposable into blocks,

then $P_{T}(G) \in \mathcal{M i n h}(G)$.

Proof. We use induction on $t$. Let $t=1$. Consider $T=\left\{v_{1}\right\} \in \mathcal{C}(G)$, with $v_{1} \in V(G)$ that belongs to at least 2 endblocks of an indecomposable component of $G$ and $H_{1}$ is decomposable into blocks. By Lemma 2.1 and Remark2.2 there exists a cutset $T^{\prime}$ that contains $v_{1}$ and no vertices of clique degree equal to 2 such that $P_{T^{\prime}}(G) \in \mathcal{M i n h}(G)$. Since $H_{1}$ is decomposable into blocks, all the non-free vertices of $H_{1}$ have clique degree equal to 2 , then $T^{\prime}=\left\{v_{1}\right\}=T$ and $P_{T}(G) \in \mathcal{M i n h}(G)$.

Let $t>1$. Consider $T=\left\{v_{1}, \ldots, v_{t}\right\} \in \mathcal{C}(G)$. The vertex $v_{1}$ belongs to at least 2 endblocks of an indecomposable component of $G$ and, by induction hypothesis, $P_{T^{\prime}}\left(H_{1}\right) \in \mathcal{M i n h}\left(H_{1}\right)$, where $T^{\prime}=\left\{v_{2}, \ldots, v_{t}\right\}$. By Lemma 2.3, $P_{T^{\prime} \cup\left\{v_{1}\right\}}(G) \in$ $\operatorname{Minh}(G)$. 
Theorem 2.5 (Algorithm: Krull Dimension of binomial edge ideals of block graphs)

- Input: A connected block graph $G$ over $[n]$.

- Output: Krull dimension of $S / J_{G}$.

1. $\operatorname{dim}:=n+1$

2. $\mathcal{G}:=\{G\}$;

3. for every graph $H \in \mathcal{G}$

4. $\mathcal{G}:=\mathcal{G} \backslash\{H\}$

5. decompose $H$ into its indecomposable subgraphs $\mathcal{I}=\left\{G_{1}, \ldots, G_{r}\right\}$;

6. remove from $\mathcal{I}$ the graphs which are blocks;

7. for every graph $G_{i} \in \mathcal{I}$

8. $\quad$ take $v \in V\left(G_{i}\right)$ such that $v$ belongs to at least 2 endblocks;

9. $\quad \operatorname{dim}:=\operatorname{dim}+\operatorname{cdeg}(v)-2$;

10. $\mathcal{G}:=\mathcal{G} \cup\left\{H_{v}\right\}$;

where $H_{v}$ denotes the graph obtained from $G_{i}$ by removing $v$ and the endblocks to which $v$ belongs.

Proof. The aim of the algorithm is to compute the Krull dimension by finding a cutset $T$ such that $P_{T}(G) \in \mathcal{M i n h}(G)$. In particular, after a finite number of steps we obtain a cutset $T=\left\{v_{1}, \ldots, v_{t}\right\}$ that fulfils the hypothesis of Theorem 2.4, and then $P_{T}(G) \in \mathcal{M i n h}(G)$. Now we explain in detail the algorithm.

Line 1 . We set $\operatorname{dim}=n+1$. This is the case when the graph $G$ is a block or is decomposable into blocks, that is $T=\emptyset$.

Line 2 . We denote by $\mathcal{G}$ the set of graphs that are to consider still.

Lines 3-4. We consider each graph $H \in \mathcal{G}$. The algorithm finishes when $\mathcal{G}$ is empty. Lines 5-6. We decompose $H$ into its indecomposable components $G_{1}, \ldots, G_{r}$. These subgraphs are the elements of the set $\mathcal{I}$. This is equivalent to do away with the vertices of clique degree 2 (see Remark 2.2). Now, by a branch and bound strategy we study each indecomposable subgraphs of $H$. We discard the blocks since their vertices are free vertices and then they do not belong to $T$.

Lines 7-8. For every subgraph $G_{i} \in \mathcal{I}$, since $G_{i}$ is indecomposable there exists a vertex $v$ that belongs to at least 2 endblocks. By Lemma 2.1. we assume $v \in T$.

Line 9. We update the Krull dimension: the number of connected components induced by $v$ in $G_{i}$ is exactly its clique degree. One of these components has been already considered, when we set $\operatorname{dim}=n+1$ in the Line 1 . Therefore, the contribute of $v$ is equal to $\operatorname{cdeg}(v)-1$ less the cardinality of the cutset, which is 1 .

Line 10. We remove from $G_{i}$ the vertex $v$ and the endblocks which contain $v$, and we add this new graph $H_{v}$ in $\mathcal{G}$, the set of graphs to consider still.

The wanted $T$ consists of all the vertices $v$ considered in Line 8. Observe that, by construction, any $v \in T$ satisfies the condition (1) of Theorem 2.4, and the condition (2) holds at the end of the algorithm, when $\mathcal{G}=\emptyset$. Moreover, the algorithm finishes after a finite number of steps: in Line 4, we remove a graph $H$ from $\mathcal{G}$ but we add some new graphs $H_{v}$ in $\mathcal{G}$ in Line 10. For any of these $H_{v}$, it holds $\left|V\left(H_{v}\right)\right|<|V(H)|$, hence after a finite number of iterations the new graphs in Line 10 will be either blocks, and then they will be discarded in Line 6 , or empty graphs. 
We highlight that the above algorithm works also for disconnected graphs: it is sufficient to set $\operatorname{dim}:=n+c$ in Line 1 , where $c$ is the number of connected components of $G$.

We are going to show that the Krull dimension of $S / J_{G}$ can be computed with a unique visit of $G$ by a recursive function, named $\operatorname{IsInT}\left(v, G\right.$, var $\left.c_{-} T\right)$. The cost of traversing a graph $G$ is $\mathcal{O}(|V(G)|+|E(G)|)$ (see [3, Section 22]). This implies that the Algorithm 2.5 can be implemented through a procedure which is linear with respect to the number of vertices and edges of $G$, without any decomposition. The function $\operatorname{IsInT}\left(v, G\right.$, var $\left.c_{-} T\right)$ constructs a $T \in \mathcal{C}(G)$ that fulfils the conditions (1) and (2) of Theorem 2.4, and then $P_{T}(G) \in \mathcal{M i n h}(G)$. For the sake of simplicity, in the following let $G$ be a tree. We recall that a vertex $v$ is called a leaf of a tree if $\operatorname{cdeg}(v)=1$.

1. $\operatorname{IsInT}\left(v, G, \operatorname{var} c_{-} T\right)$

2. if $v$ is a leaf then

3. return 0

4. else

5. $\quad$ degree $:=\operatorname{cdeg}(v)$;

6. $\quad$ childrenInT $:=0$

7. for every children $w$ of $v$ childrenInT $:=$ childrenInT $+\operatorname{IsInT}\left(w, G, c_{-} T\right) ;$

degree $:=$ degree - chidrenInT;

if degree $>2$ then

$c_{T}:=c_{T}+$ degree -2 ;

return 1

else

return 0

Even if the algorithm works for any undirected tree, we assign an orientation given by the visit of the tree itself: the children of a given vertex are its adjacent vertices that have not been visited yet. The purpose of $\operatorname{IsInT}\left(v, G, c_{-} T\right)$ is twofold: on one side, starting from any vertex $v \in V(G)$, it checks if $v$ belongs to $T$ and in this case it returns 1 , otherwise 0 , on the other side it computes $c(T)-|T|$, which is saved in $c_{T}$. For a vertex $v$ being in $T$ depends on its children that are in $T$, and on its degree. The latter is given by the initial degree less the number of children of $v$ that are in $T$ (Line 9). In particular, $v \in T$ if at least 2 of its children are not in $T$ and its degree is greater than 2 (Line 10).

To compute the Krull dimension of $S / J_{G}$, it is sufficient to call the function $\operatorname{IsInT}\left(v, G, c_{-} T\right)$, where $v$ is any vertex of $G$ and $c_{T}$ is a global variable set to 1 , and then $\operatorname{dim} S / J_{G}=n+c_{T}$.

We have implemented this procedure for trees using CoCoA version 4.7 and it is freely downloadable on 13 .

\section{REGULARITY BOUNDS FOR BINOMIAL EDGE IDEALS OF BLOCK GRAPHS}

The main result of this section is the lower bound for the Castelnuovo-Mumford regularity of binomial edge ideals of block graphs (Theorem 3.6). To reach our result, we compute the regularity and the superextremal Betti numbers of special block graphs, called flower graphs.

Let $M$ be a finitely generated graded $S$-module. A Betti number $\beta_{i, i+j}(M) \neq 0$ is called extremal if $\beta_{k, k+\ell}=0$ for all pairs $(k, \ell) \neq(i, j)$, with $k \geq i, \ell \geq j$. Let 
$q=\operatorname{reg} M$ and $p=\operatorname{projdim} M$, then there exist unique numbers $i$ and $j$ such that $\beta_{i, i+q}(M)$ and $\beta_{p, p+j}(M)$ are extremal Betti numbers. We call them the distinguished extremal Betti numbers of $M$. Let $k$ be the maximal integer $j$ such that $\beta_{i, j} \neq 0$ for some $i$. It is clear that $\beta_{i, k}(M)$ is an extremal Betti number for all $\mathrm{i}$ with $\beta_{i, k} \neq 0$, and that there is at least one such $i$. These Betti numbers are distinguished by the fact that they are positioned on the diagonal $\{(i, k-1) \mid i=0, \ldots, k\}$ in the Betti diagram, and that all Betti numbers on the right lower side of the diagonal are zero. The Betti numbers $\beta_{i, k}$, for $i=0, \ldots, k$, are called superextremal, regardless of whether they are zero or not. We refer the reader to [7, Chapter 11] for further details.

Let $G$ be a graph. We denote by $i(G)$ the number of inner vertices of $G$ and by $f(G)$ the number of free vertices of $G$.

Definition 3.1 A flower graph $F_{h, k}(v)$ is a connected block graph constructed by joining $h$ copies of the cycle graph $C_{3}$ and $k$ copies of the bipartite graph $K_{1,3}$ with a common vertex $v$, where $v$ is one of the free vertices of $C_{3}$ and of $K_{1,3}$, and $\operatorname{cdeg}(v) \geq 3$.

We observe that any flower graph $F_{h, k}(v)$ has $2 h+3 k+1$ vertices and $3(h+k)$ edges. The clique degree of $v$ is given by $h+k$, and the number of inner vertices is $i\left(F_{h, k}(v)\right)=k+1$ and all of them are cutpoints for $F_{h, k}(v)$. When it is unnecessary to make explicit the parameters $h$ and $k$, we refer to $F_{h, k}(v)$ as $F(v)$.

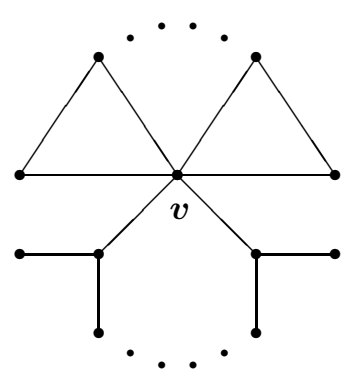

Figure 1. A flower graph $F_{h, k}(v)$

Remark 3.2. Let $G$ be a flower graph $F(v)$. By the result [18, Corollary 1.5], $G=J_{G^{\prime}} \cap Q_{v}$ where $G^{\prime}$ is the graph obtained from $G$ by connecting all the vertices adjacent to $v$, and $Q_{v}=\bigcap_{T \in \mathcal{C}(G), v \in T} P_{T}(G)$. We observe that in this case $Q_{v}=$ $\left(x_{v}, y_{v}\right)+J_{G^{\prime \prime}}$, where $G^{\prime \prime}$ is obtained from $G$ by removing $v$, and then

$$
J_{G}=J_{G^{\prime}} \cap\left(\left(x_{v}, y_{v}\right)+J_{G^{\prime \prime}}\right) .
$$

Before stating the distinguished extremal Betti numbers of the binomial edge ideal of a flower graph, we need the following remark.

Remark 3.3. Let $G$ be a disconnected block graph with $G_{1}, \ldots, G_{r}$ its connected components. If all the $G_{j}$ have precisely one extremal Betti number, $\beta_{n_{j}-1, n_{j}+i\left(G_{j}\right)}\left(S_{j} / J_{G_{j}}\right)$, 
for any $j=1, \ldots, r$, with $S_{j}=K\left[x_{i}, y_{i}\right]_{i \in V\left(G_{j}\right)}$ and $n_{j}=\left|V\left(G_{j}\right)\right|$, then $S / J_{G}$ has precisely one extremal Betti number and it is given by

$$
\beta_{n-r, n+i(G)}\left(S / J_{G}\right)=\prod_{j=1}^{r} \beta_{n_{j}-1, n_{j}+i\left(G_{j}\right)}\left(S_{j} / J_{G_{j}}\right) .
$$

Theorem 3.4 Let $G$ be a flower graph $F(v)$. The following are extremal Betti numbers of $S / J_{G}$ :

(i) $\beta_{n-1, n+i(G)}\left(S / J_{G}\right)=f(G)-1$;

(ii) $\beta_{n-\operatorname{cdeg}(v)+1, n+i(G)}\left(S / J_{G}\right)=1$.

In particular, they are the only non-zero superextremal Betti numbers.

Proof. The fact (1) is proved in 9, Theorem 2.2]. As regards (2), we focus on the cutpoint $v$ of $G$. Thanks to the decomposition quoted in Remark 3.2, we consider the following exact sequence

$$
0 \longrightarrow S / J_{G} \longrightarrow S / J_{G^{\prime}} \oplus S /\left(\left(x_{v}, y_{v}\right)+J_{G^{\prime \prime}}\right) \longrightarrow S /\left(\left(x_{v}, y_{v}\right)+J_{H}\right) \longrightarrow 0
$$

where $G^{\prime}$ and $G^{\prime \prime}$ are described in Remark [3.2 and $H$ is obtained from $G^{\prime}$ by removing $v$. We observe that $G^{\prime}$ and $H$ are block graphs satisfying [9, Theorem 2.4 (b)], with $i\left(G^{\prime}\right)=i(H)=i(G)-1$, and then $\operatorname{reg} S / J_{G^{\prime}}=\operatorname{reg} S /\left(\left(x_{v}, y_{v}\right)+J_{H}\right)=$ $i(G)$. The graph $G^{\prime \prime}$ has $\operatorname{cdeg}(v)$ connected components $G_{1}, \ldots, G_{\operatorname{cdeg}(v)}$ : all of them are either $K_{2}$ or paths of length 2 , namely $P_{2}$. The latter are decomposable into two $K_{2}$ and it holds $\operatorname{reg} S^{\prime} / J_{P_{2}}=2=i\left(P_{2}\right)+1$, with $S^{\prime}=K\left[x_{i}, y_{i}\right]_{i \in V\left(P_{2}\right)}$. Then, by [9, Theorem 2.4 (b)] and since the ring $S /\left(\left(x_{v}, y_{v}\right)+J_{G^{\prime \prime}}\right)$ is the tensor product of $S_{j} / J_{G_{j}}$, with $j=1, \ldots, \operatorname{cdeg}(v)$ and $S_{j}=K\left[x_{i}, y_{i}\right]_{i \in V\left(G_{j}\right)}$, we have

$$
\operatorname{reg} \frac{S}{\left(x_{v}, y_{v}\right)+J_{G^{\prime \prime}}}=\sum_{j=1}^{\operatorname{cdeg}(v)} \operatorname{reg} \frac{S_{j}}{J_{G_{j}}}=\sum_{j=1}^{\operatorname{cdeg}(v)}\left(i\left(G_{j}\right)+1\right)=i(G)-1+\operatorname{cdeg}(v) \text {. }
$$

We get the following bound on the regularity of $S / J_{G}$

$$
\begin{aligned}
\operatorname{reg} S / J_{G} & \leq \max \left\{\operatorname{reg} \frac{S}{J_{G^{\prime}}}, \operatorname{reg} \frac{S}{\left(x_{v}, y_{v}\right)+J_{G^{\prime \prime}}}, \operatorname{reg} \frac{S}{\left(x_{v}, y_{v}\right)+J_{H}}+1\right\} \\
& =\max \{i(G), i(G)-1+\operatorname{cdeg}(v), i(G)+1\} \\
& =i(G)-1+\operatorname{cdeg}(v) .
\end{aligned}
$$

By [4, Theorem 1.1], the depth of $S / J_{G}$ for any block graph $G$ over $[n]$ is equal to $n+c$, where $c$ is the number of connected components of $G$. Since we know the depth of all quotient rings involved in (5) and by Auslander-Buchsbaum formula, we get $\operatorname{proj} \operatorname{dim} S / J_{G}=\operatorname{proj} \operatorname{dim} S / J_{G^{\prime}}=\operatorname{proj} \operatorname{dim} S /\left(\left(x_{v}, y_{v}\right)+J_{H}\right)-1=n-1$, and $\operatorname{proj} \operatorname{dim} S /\left(\left(x_{v}, y_{v}\right)+J_{G^{\prime \prime}}\right)=n-\operatorname{cdeg}(v)+1$.

Let $j>i(G)$, then

$$
T_{m, m+j}\left(S / J_{G^{\prime}}\right)=T_{m, m+j}\left(S /\left(\left(x_{v}, y_{v}\right)+J_{H}\right)\right)=0 \quad \text { for any } m,
$$

and

$$
T_{m, m+j}\left(S /\left(\left(x_{v}, y_{v}\right)+J_{G^{\prime \prime}}\right)\right)=0 \quad \text { for any } m>n-\operatorname{cdeg}(v)+1,
$$

where $T_{m, m+j}^{S}(M)$ stands for $\operatorname{Tor}_{m, m+j}^{S}(M, K)$ for any $S$-module $M$, and $S$ is omitted if it is clear from the context. Of course, all the above Tor modules $T_{m, m+j}(-)$ are zero when $j>i(G)-1+\operatorname{cdeg}(v)$. 
Therefore, for $m=n-\operatorname{cdeg}(v)+1$ and $j=i(G)-1+\operatorname{cdeg}(v)$ we obtain the following long exact sequence

$$
\begin{aligned}
\cdots & \rightarrow T_{m+1, m+1+(j-1)}\left(S /\left(\left(x_{v}, y_{v}\right)+J_{H}\right)\right) \rightarrow T_{m, m+j}\left(S / J_{G}\right) \rightarrow \\
& T_{m, m+j}\left(S / J_{G^{\prime}}\right) \oplus T_{m, m+j}\left(S /\left(\left(x_{v}, y_{v}\right)+J_{G^{\prime \prime}}\right)\right) \rightarrow \\
& T_{m, m+j}\left(S /\left(\left(x_{v}, y_{v}\right)+J_{H}\right)\right) \rightarrow \cdots
\end{aligned}
$$

In view of the above, all the functors on the left of $T_{m, m+j}\left(S / J_{G}\right)$ in the long exact sequence are zero, and $T_{m, m+j}\left(S / J_{G^{\prime}}\right)=T_{m, m+j}\left(S /\left(\left(x_{v}, y_{v}\right)+J_{H}\right)\right)=0$ too. It follows

$$
T_{m, m+j}\left(S / J_{G}\right) \cong T_{m, m+j}\left(S /\left(\left(x_{v}, y_{v}\right)+J_{G^{\prime \prime}}\right)\right) .
$$

It means that

$$
\beta_{n-\operatorname{cdeg}(v)+1, n+i(G)}\left(S / J_{G}\right)=\beta_{n-\operatorname{cdeg}(v)+1, n+i(G)}\left(S /\left(\left(x_{v}, y_{v}\right)+J_{G^{\prime \prime}}\right)\right) .
$$

We observe that

$$
T_{m, m+j}^{S}\left(S /\left(\left(x_{v}, y_{v}\right)+J_{G^{\prime \prime}}\right)\right) \cong T_{m-2, m-2+j}^{S^{\prime \prime}}\left(S^{\prime \prime} / J_{G^{\prime \prime}}\right)
$$

where $S^{\prime \prime}=S /\left(x_{v}, y_{v}\right)$. Since all the connected components $G_{1}, \ldots, G_{\operatorname{cdeg}(v)}$ of $G^{\prime \prime}$ are either a $K_{2}$ or a path of length 2 , the quotient rings $S_{j} / J_{G_{j}}$ have an unique extremal Betti number $\beta_{n_{j}-1, n_{j}+i\left(G_{j}\right)}\left(S_{j} / J_{G_{j}}\right)$, for $j=1, \ldots, \operatorname{cdeg}(v)$ and $n_{j}=$ $\left|V\left(G_{j}\right)\right|$, which is equal to 1 . Therefore, by Remark 3.3 we have

$$
\beta_{m-2, m-2+j}\left(S^{\prime \prime} / J_{G^{\prime \prime}}\right)=\prod_{j=1}^{\operatorname{cdeg}(v)} \beta_{n_{j}-1, n_{j}+i\left(G_{j}\right)}\left(S_{j} / J_{G_{j}}\right)=1 .
$$

Observe that for $m=n-\operatorname{cdeg}(v)+1$ and $j=i(G)-1+\operatorname{cdeg}(v)$ we get that $m+j=n+i(G)$ is the maximal integer such that $\beta_{i, m+j}\left(S / J_{G}\right) \neq 0$ for some $i$. We want to prove that $\beta_{i, n+i(G)} \neq 0$, only for $i=n-\operatorname{cdeg}(v)+1$ and $i=n-1$. Let $i$ be an integer such that $\beta_{i, n+i(G)} \neq 0$. Since $\operatorname{proj} \operatorname{dim} S / J_{G}=n-1$ and $\operatorname{reg} S / J_{G} \leq i(G)+\operatorname{cdeg}(v)-1$, we have to examine $n-\operatorname{cdeg}(v)+1 \leq i \leq n-1$. Consider the following long exact sequence

$$
\begin{aligned}
\cdots & \rightarrow T_{i+1, n+i(G)}\left(\frac{S}{\left(x_{v}, y_{v}\right)+J_{H}}\right) \rightarrow T_{i, n+i(G)}\left(\frac{S}{J_{G}}\right) \rightarrow \\
& T_{i, n+i(G)}\left(\frac{S}{J_{G^{\prime}}}\right) \oplus T_{i, n+i(G)}\left(\frac{S}{\left(x_{v}, y_{v}\right)+J_{G^{\prime \prime}}}\right) \rightarrow \\
& T_{i, n+i(G)}\left(\frac{S}{\left(x_{v}, y_{v}\right)+J_{H}}\right) \rightarrow \cdots
\end{aligned}
$$

If $n-\operatorname{cdeg}(v)+1<i<n-1$, since $i>\operatorname{proj} \operatorname{dim} S /\left(\left(x_{v}, y_{v}\right)+J_{G^{\prime \prime}}\right)$ and $n+$ $i(G)-i>\operatorname{reg} S / J_{G^{\prime}}, \operatorname{reg} S /\left(\left(x_{v}, y_{v}\right)+J_{H}\right)$, it holds $\operatorname{Tor}_{i, n+i(G)}(M)=0$, for $M \in$ $\left\{S / J_{G^{\prime}}, S /\left(\left(x_{v}, y_{v}\right)+J_{G^{\prime \prime}}\right), S /\left(\left(x_{v}, y_{v}\right)+J_{H}\right)\right\}$, and then we can conclude that also $\operatorname{Tor}_{i, n+i(G)}\left(S / J_{G}\right)=0$.

An immediate consequence of the proof of the Theorem 3.4 is the regularity of any flower graphs $F(v)$, that depends only on the clique degree of $v$ and the number of inner vertices of $F(v)$.

Corollary 3.5 Let $F(v)$ be a flower graph, then

$$
\operatorname{reg} S / J_{F(v)}=i(F(v))+\operatorname{cdeg}(v)-1 .
$$




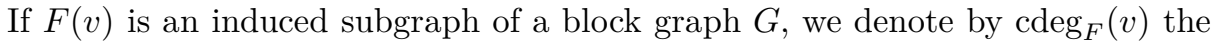
clique degree of $v$ in $F(v)$. Note that if $F(v)$ is the maximal flower induced subgraph of $G$ and all the blocks of $G$ containing $v$ are $C_{3}$ or $K_{1,3}$, then $\operatorname{cdeg}_{F}(v)=\operatorname{cdeg}(v)$, otherwise $\operatorname{cdeg}_{F}(v)<\operatorname{cdeg}(v)$.

Theorem 3.6 Let $G$ be an indecomposable block graph and let $F(v)$ be an induced subgraph of $G$. Then

$$
\operatorname{reg} S / J_{G} \geq i(G)+\operatorname{cdeg}_{F}(v)-1
$$

Proof. We use induction on the number of blocks of $G$ that are not in $F(v)$. If $G=$ $F(v)$, the statement follows from Corollary 3.5. Suppose now $G$ contains properly $F(v)$ as induced subgraph. Since $G$ is connected, there exists an endblock $B$ of $G$ and a subgraph $G^{\prime}$ of $G$ such that $G=G^{\prime} \cup B, G^{\prime}$ contains $F(v)$ as induced subgraph, $V\left(G^{\prime}\right) \cap V(B)=\{w\}$, and all the blocks containing $w$ are endblocks, except for the one that is in $G^{\prime}$. Since $G$ is assumed to be indecomposable, $\operatorname{cdeg}(w) \geq 3$. If $\operatorname{cdeg}(w)=3$, then $G^{\prime}$ is decomposable into $G_{1} \cup G_{2}$, and $\operatorname{reg} S / J_{G^{\prime}}=\operatorname{reg} S / J_{G_{1}}+$ $\operatorname{reg} S / J_{G_{2}}$. We may suppose that $G_{1}$ contains $F(v)$, and then $i\left(G_{1}\right)=i(G)-1$, but $\operatorname{cdeg}_{F}(v)$ is still the same. Whereas, $G_{2}$ is a block and $\operatorname{reg} S / J_{G_{2}}=1$. Then by using induction, we may assume that $\operatorname{reg} S / J_{G_{1}} \geq i(G)+\operatorname{cdeg}_{F}(v)-2$. Therefore,

$$
\operatorname{reg} S / J_{G^{\prime}}=\operatorname{reg} S / J_{G_{1}}+\operatorname{reg} S / J_{G_{2}} \geq i(G)+\operatorname{cdeg}_{F}(v)-1 .
$$

If $\operatorname{cdeg}(w)>3$, then $i\left(G^{\prime}\right)=i(G)$ and $\operatorname{cdeg}_{F}(v)$ is still the same. Then, by using induction on the number of blocks of $G$, we may assume $\operatorname{reg} S / J_{G^{\prime}} \geq i(G)+$ $\operatorname{cdeg}_{F}(v)-1$. By [14, Corollay 2.2] of Matsuda and Murai, one have that

$$
\operatorname{reg} S / J_{G} \geq \operatorname{reg} S / J_{G^{\prime}}
$$

and then $\operatorname{reg} S / J_{G} \geq i(G)+\operatorname{cdeg}_{F}(v)-1$, as desired.

Definition 3.7 Let $G$ be a block graph. If $G$ has no flower graphs as induced subgraphs then $G$ is called flower-free.

We are ready to state the following bound for the regularity for any binomial edge ideal of block graphs.

Corollary 3.8 Let $G$ be a connected block graph which is not an isolated vertex.

(i) If $G$ is a flower-free graph, then $\operatorname{reg} S / J_{G}=i(G)+1$.

(ii) If $G$ contains $r \geq 1$ flower graphs $F_{1}\left(v_{1}\right), \ldots, F_{r}\left(v_{r}\right)$ as induced subgraphs, then $\operatorname{reg} S / J_{G} \geq i(G)+\max _{i=1, \ldots, r}\left\{\operatorname{cdeg}_{F_{i}}\left(v_{i}\right)\right\}-1$.

Proof. (1) If $G$ is indecomposable, by [9, Theorem 2.4], the result follows. Otherwise, suppose $G$ is decomposable into indecomposable graphs $G_{1}, \ldots, G_{r}$. Observe that if $v$ is an inner vertex in $G$ then either $\{v\}=G_{i} \cap G_{j}$ for some $i \neq j$ and it is a free vertex in $G_{i}$ and $G_{j}$, or it belongs to an unique $G_{i}$ and it is an inner vertex of $G_{i}$. The former are exactly $r-1$. In fact, if we consider the graph $T$, with vertices $V(T)=\left\{G_{1}, \ldots, G_{r}\right\}$ and edges $E(T)=\left\{\left\{G_{i}, G_{j}\right\}: G_{i} \cap G_{j} \neq \emptyset\right\}$ we observe that 
$T$ is a tree and $|E(T)|=r-1$. Hence

$$
i(G)=r-1+\sum_{i=1}^{r} i\left(G_{i}\right) .
$$

By Proposition 1.2 and [9, Theorem 2.4], we get

$$
\operatorname{reg} S / J_{G}=\sum_{i=1}^{r} \operatorname{reg} S / J_{G_{i}}=\sum_{i=1}^{r}\left(i\left(G_{i}\right)+1\right)=i(G)+1 .
$$

(2) It is an immediate consequence of [14, Corollary 2.2] and Theorem 3.6.

Example 3.9. Let $G$ be the graph in Figure 2, It contains 2 flower graphs as induced subgraphs: $F_{2,1}\left(v_{1}\right)$ and $F_{3,1}\left(v_{2}\right)$. By Corollary 3.8 we have reg $S / J_{G} \geq$ $2+\max \{3,4\}-1=5$, whereas the length of the longest induced path in $G$ is 3 and the number of maximal cliques of $G$ is 6 . Also using the upper bound proved in [11, we get reg $S / J_{G} \leq 6$. By means of a computation in CoCoA, reg $S / J_{G}=5$, it means the lower bound given in Corollary 3.8 is sharp. We observe that $G$ is the graph with the minimum number of vertices such that $S / J_{G}$ has 3 non-zero superextremal Betti numbers.

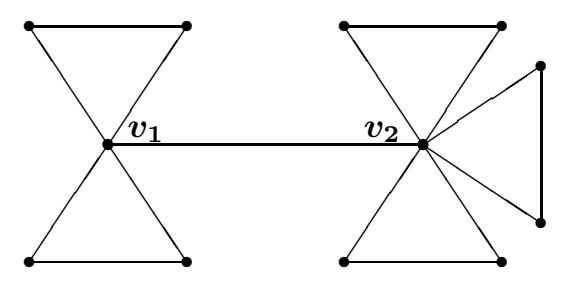

Figure 2. A graph $G$ such that $\operatorname{reg} S / J_{G}=i(G)+$ $\max _{i=1,2}\left\{\operatorname{cdeg}_{F_{i}}\left(v_{i}\right)\right\}-1$.

Example 3.9 encourages us to follow up with an algorithm to compute the regularity of binomial edge ideal of block graphs, and it will be the content of the section 4

The bound exhibited in Corollary 3.8 can be improved for block graphs with several flowers $F_{i}\left(v_{i}\right)$ with the vertices $v_{i}$ far enough from each other. In particular, let $H$ be an induced subgraph of $G$ and suppose $H$ is decomposable into $H_{1}, \ldots, H_{r}$ such that any $H_{i}$ contains a flower graph $F_{i}\left(v_{i}\right)$ as an induced subgraph for $i=$ $1, \ldots, r$. Then $\operatorname{reg} J_{G} \geq \operatorname{reg} J_{H}=\sum_{i=1}^{r} \operatorname{reg} J_{H_{i}}$, which could be better than the one provided in Corollary 3.8 .

\section{How to compute the Castelnuovo-Mumford Regularity of Block GRAPHS}

In this section we provide an efficient method to compute the CastelnuovoMumford regularity for $S / J_{G}$ when $G$ is a block graph. 
Definition 4.1 Let $G$ be a block graph and $F(v)$ be a flower graph that is an induced subgraph of $G . F(v)$ is called an end-flower of $G$ if $G=G_{1} \cup \ldots \cup G_{c}$, where $c=\operatorname{cdeg}(v)$, and such that $G_{i} \cap G_{j}=\{v\}$, for all $1 \leq i<j \leq c$, and $G_{2}, \ldots G_{c}$ are flower-free graphs.

Theorem 4.2 Let $G$ be a block graph, $v_{1}, \ldots, v_{r} \in V(G)$,

$$
H_{j}=G \backslash\left\{v_{1}, \ldots, v_{j}\right\}
$$

for $j=1, \ldots, r$, and $H_{0}=G$. If

(i) $F\left(v_{j}\right)$ is an end-flower for $H_{j-1}$, for all $j=1, \ldots, r$,

(ii) $H_{r}$ is flower-free,

then

$$
\operatorname{reg} S / J_{G}=\operatorname{reg} S / J_{H_{r}}=c+i\left(H_{r}\right)
$$

where $c$ is the number of connected components of $H_{r}$ which are not isolated vertices.

Proof. First of all, observe that the equality reg $S / J_{H_{r}}=c+i\left(H_{r}\right)$ in the statement is an immediate consequence of Corollary [3.8 (1).

To prove $\operatorname{reg} S / J_{G}=\operatorname{reg} S / J_{H_{r}}$, we make induction on

$$
f=\mid\{v \in V(G) \mid F(v) \text { is an induced subgraph of } G\} \mid .
$$

If $f=0$, that is $G$ is a flower-free graph, then the assertion follows by Corollary 3.8 (1). Let $f=1$ and $v$ be such that $F(v)$ is an induced subgraph of $G$. Consider the exact sequence

$$
0 \longrightarrow S / J_{G} \longrightarrow S / J_{G^{\prime}} \oplus S /\left(\left(x_{v}, y_{v}\right)+J_{G^{\prime \prime}}\right) \longrightarrow S /\left(\left(x_{v}, y_{v}\right)+J_{H}\right) \longrightarrow 0
$$

where $G^{\prime}, G^{\prime \prime}$, and $H$ are as described in Remark 3.2. We observe that $G^{\prime}, G^{\prime \prime}$, and $H$ are flower-free. Hence

$$
\operatorname{reg} S / J_{G^{\prime}}=\operatorname{reg} S / J_{H}=i\left(G^{\prime}\right)+1=i(G)-1+1=i(G) .
$$

Moreover, removing the vertex $v$ from $G$ we obtain $G^{\prime \prime}$ and $\operatorname{reg} S / J_{G^{\prime \prime}}$ is

$$
\sum_{j=1}^{c} \operatorname{reg} S / J_{G_{j}}=\sum_{j=1}^{c}\left(i\left(G_{j}\right)+1\right)=\sum_{j=1}^{\operatorname{cdeg}_{F}(v)}\left(i\left(G_{j}\right)+1\right)+\sum_{k=1}^{c^{\prime}}\left(i\left(G_{k}\right)+1\right)
$$

where $G_{1}, \ldots, G_{c}$ are the connected components of $G^{\prime \prime}$, and $\left\{v, w_{k}\right\}$ are maximal cliques in $G$ with $w_{k}$ a free vertex of $G_{k}$, and $\left|V\left(G_{k}\right)\right| \geq 2$, for $k=1, \ldots, c^{\prime}$. Observe that, for $j=1, \ldots, \operatorname{cdeg}_{F}(v)$, all the inner vertices of $G$ that belong to $G_{j}$ are inner vertices also in $G^{\prime \prime}$. Whereas, for $k=1, \ldots, c^{\prime}$, the $w_{k}$ are inner vertices in $G$ but not in $G^{\prime \prime}$, and all the other inner vertices of $G$ that belong to $G_{k}$ are inner vertices also in $G^{\prime \prime}$. Hence, removing $v$ from $G$, we have $c^{\prime}+1$ less inner vertices in $G^{\prime \prime}$ with respect to $G$, that are all the $w_{k}$ and $v$, but this is compensated by the formula $\sum_{k=1}^{c^{\prime}}\left(i\left(G_{k}\right)+1\right)=c^{\prime}+\sum_{k=1}^{c^{\prime}} i\left(G_{k}\right)$. Hence

$$
\operatorname{reg} S / J_{G^{\prime \prime}}=i(G)+\operatorname{cdeg}_{F}(v)-1 .
$$

Since $\operatorname{cdeg}_{F}(v) \geq 3$,

$$
\operatorname{reg} S / J_{G^{\prime}}, \operatorname{reg} S /\left(\left(x_{v}, y_{v}\right)+J_{H}\right)<\operatorname{reg} S /\left(\left(x_{v}, y_{v}\right)+J_{G^{\prime \prime}}\right)
$$

and then $\operatorname{reg} S / J_{G}=\operatorname{reg} S /\left(\left(x_{v}, y_{v}\right)+J_{G^{\prime \prime}}\right)$. 
Let $f>1$. Let $v_{1}, \ldots, v_{r} \in V(G)$ be a sequence that fulfills (1) and (2). Consider the exact sequence (6), with $v=v_{1}$. Observe that the sequence $v_{2}, \ldots, v_{r}$ satisfies (1) and (2) for $G^{\prime}, G^{\prime \prime}$, and $H$ and, since they have less than $f$ flower graphs as induced subgraphs, by induction hypothesis their regularity is given by the sum of the regularity of the connected components induced by $v_{2}, \ldots, v_{r}$.

Let $G_{1}, \ldots, G_{m}$ be the connected components induced by $v_{2}, \ldots, v_{r}$ in $G$. One of them contains $v_{1}$, suppose $G_{1}$, and then it is not flower-free, whereas the others are flower-free. The connected components induced by $v_{2}, \ldots, v_{r}$ in $G^{\prime}$ and $H$ are $G_{1}^{\prime}, G_{2}, \ldots, G_{m}$ and $G_{1}^{\prime} \backslash\left\{v_{1}\right\}, G_{2}, \ldots, G_{m}$, respectively, where $G_{1}^{\prime}$ denotes the graph obtained from $G_{1}$ by connecting all the vertices adjacent to $v_{1}$. We get

$$
\operatorname{reg} S / J_{G^{\prime}}=\operatorname{reg} S / J_{H}=\operatorname{reg} S / J_{G_{1}^{\prime}}+\sum_{i=2}^{m} \operatorname{reg} S / J_{G_{i}} .
$$

Whereas, the connected components induced by $v_{2}, \ldots, v_{r}$ in $G^{\prime \prime}$ are the connected components of $G_{1} \backslash\left\{v_{1}\right\}$ and $G_{2}, \ldots, G_{m}$, and then

$$
\operatorname{reg} S / J_{G^{\prime \prime}}=\operatorname{reg} S / J_{G_{1} \backslash\left\{v_{1}\right\}}+\sum_{i=2}^{m} \operatorname{reg} S / J_{G_{i}} .
$$

Since

$$
\operatorname{reg} S / J_{G_{1}^{\prime}}=i\left(G_{1}\right)<i\left(G_{1}\right)+\operatorname{cdeg}_{F}\left(v_{1}\right)-1=\operatorname{reg} S / J_{G_{1} \backslash\left\{v_{1}\right\}},
$$

where the last equality follows from the same arguments of above and $\operatorname{cdeg}_{F}\left(v_{1}\right)$ denotes the clique degree of $v_{1}$ in $F\left(v_{1}\right)$, with $F\left(v_{1}\right)$ seen as induced subgraph of $G_{1}$. Since $F\left(v_{1}\right)$ is an end-flower and $\operatorname{cdeg}_{F}\left(v_{1}\right) \geq 3$ in $G$, it follows $\operatorname{cdeg}_{F}\left(v_{1}\right) \geq 2$ in $G_{1}$. Observe that, when $\operatorname{cdeg}_{F}\left(v_{1}\right)=2$ in $G_{1}, G_{1}$ is flower-free and it is easy to see that the equality $\operatorname{reg} S / J_{G_{1} \backslash\left\{v_{1}\right\}}=i\left(G_{1}\right)+\operatorname{cdeg}_{F}\left(v_{1}\right)-1$ is still true. Then

$$
\operatorname{reg} S / J_{G^{\prime}}, \operatorname{reg} S /\left(\left(x_{v}, y_{v}\right)+J_{H}\right)<\operatorname{reg} S /\left(\left(x_{v}, y_{v}\right)+J_{G^{\prime \prime}}\right)
$$

and the assertion is proved.

The Theorem 4.2 suggests a recursive way to compute the regularity of $S / J_{G}$ when $G$ is a block graph.

1. ComputeRegularity $(G)$

2. if $G$ is flower-free and is not an isolated vertex

3. return $i(G)+1$

4. else

5. $\quad \operatorname{reg}:=0$;

6. $\quad$ pinpoint an end-flower $F(v)$ of $G$;

7. $\quad$ remove $v$ from $G$;

8. for every connected component $G_{i}$ induced by $v$ in $G$

9. $\quad \operatorname{reg}:=\operatorname{reg}+$ ComputeRegularity $\left(G_{i}\right)$;

10. return reg

By means of an unique block graph traversal, that is linear with respect to the number of vertices and edges of $G$ (see [3, Section 22]), one get the regularity of $S / J_{G}$. This allows to compute the regularity of $S / J_{G}$ also for those block graphs with a large number of vertices, and then for those binomial edge ideals with a large number of variables, for which the algebraic softwares, as CoCoA [2] and Macaulay2 [6], fail. 


\section{REFERENCES}

[1] F. Chaudhry, A. Dokuyucu, R. Irfan, On the binomial edge ideals of block graphs, An. Ştiinţ. Univ. "Ovidius" Constanţa Ser. Mat.An. 24 (2016) 149-158.

[2] CoCoATeam, CoCoA: a system for doing Computations in Commutative Algebra, Available at http://cocoa.dima.unige.it

[3] T. H. Cormen, C. E. Leiserson, R. L. Rivest, C. Stein, Introduction to Algorithms, Second Edition, MIT Press and McGraw-Hill (2001).

[4] V. Ene, J. Herzog, T. Hibi, Cohen-Macaulay binomial edge ideals, Nagoya Math. J. 45 (2011) $57-68$.

[5] V. Ene, A. Zarojanu, On the regularity of binomial edge ideals, Math. Nachr. 45 (2015) $19-24$.

[6] D. R. Grayson, M. E. Stillman, Macaulay2, a software system for research in algebraic geometry, Available at http://www.math.uiuc.edu/Macaulay2/

[7] J. Herzog, T. Hibi, Monomial Ideals, Grad. Texts in Math. 260, Springer, London (2010).

[8] J. Herzog, T. Hibi, F. Hreinsdottir, T. Kahle, J. Rauh, Binomial edge ideals and conditional independence statements, Adv. in Appl. Math. 45 (2010) 317-333.

[9] J. Herzog, G. Rinaldo, On the extremal Betti numbers of binomial edge ideals of block graphs, Electron. J. Combin. 25(1) (2018) 1-10.

[10] A. V. Jayanthan, N. Narayanan, B. V. Raghavendra Rao, Regularity of binomial edge ideals of certain block graphs, Accepted in Proc. Indian Acad. Sci. Math. Sci. (2018).

[11] A. V. Jayanthan, N. Narayanan, B. V. Raghavendra Rao, An upper bound for the regularity of binomial edge ideals of trees, Accepted in J. Algebra Appl. (2018).

[12] D. Kiani, S. Saeedi Madani, The Castelnuovo-Mumford regularity of binomial edge ideals, Combin. Theory Ser. A 139 (2016) 80-86.

[13] C. Mascia, G. Rinaldo, A linear time algorithm to compute the Krull dimension of binomial edge ideal of trees (2018) http://www.giancarlorinaldo.it/krulldimtrees

[14] K. Matsuda, S. Murai, Regularity bounds for binomial edge ideals, Commut. Algebra 5 (2013) $141-149$.

[15] M. Ohtani, Graphs and ideals generated by some 2-minors, Comm. Algebra 39 (2011) 905917.

[16] A. Rauf, G. Rinaldo, Construction of Cohen-Macaulay binomial edge ideals, Comm. Algebra 42.1 (2014) pp. 238-252.

[17] G. Rinaldo, Cohen-Macaulay binomial edge ideals of small deviation, Bull. Math. Soc. Sci. Math. Roumanie 56(104) No. 4 (2013) 497-503.

[18] G. Rinaldo, Cohen-Macaulay binomial edge ideals of cactus graphs, J. Algebra Appl. 18, No. 04 (2019) 1-18.

[19] S. Saeedi Madani, D. Kiani, Binomial edge ideals of graphs, Electron. J. Combin. 19 (2012) Paper \#P44.

[20] S. Saeedi Madani, D. Kiani, On the binomial edge ideal of a pair of graphs, Electron. J. Combin. 20(1) (2013) Paper \# P48.

[21] S. Saeedi Madani, D. Kiani, Binomial edge ideals of regularity 3, J. Algebra 515 (2018) $157-172$.

University of Trento

E-mail address: carla.mascia@unitn.it

UNIVERSITY OF TRENTO

E-mail address: giancarlo.rinaldo@unitn.it 